\title{
What about the other two-thirds of the cardiac cycle?
}

\author{
Barry A. Finegan, MB
}

Published online: 28 February 2009

(c) Canadian Anesthesiologists' Society 2009

Diastolic dysfunction is a relatively new clinical diagnosis being made in patients where the observed echocardiographic findings are deemed to be consistent with abnormalities of left ventricular (LV) filling. Left ventricular filling is dependent on the complex interaction of three processes: LV relaxation, LV compliance, and left atrial (LA) contraction. ${ }^{1}$ Left ventricular relaxation is an active process involving the uptake of $\mathrm{Ca}^{++}$into the sarcoplasmic reticulum by the sarcoendoplasmic reticulum $\mathrm{Ca}^{++}$ATPase pump and removal of $\mathrm{Ca}^{++}$from the cell by way of the sodium-calcium exchanger. Sarcoendoplasmic reticulum $\mathrm{Ca}^{++}$ATPase pump expression and activity is reduced in the cardiomyocyte (and diastolic function is altered) with the onset of senescence, hypertrophy, and heart failure. Left ventricular compliance is a measure of the relationship between LV volume and LV pressure development during $\mathrm{LV}$ filling ( $\mathrm{dV} / \mathrm{dp})$. Left ventricular compliance is influenced by: 1) the presence of ventricular hypertrophy; 2) the functional state of the right ventricle (direct diastolic ventricular interaction), since both ventricles share the septum as a common wall; and 3 ) the properties of the pericardium. ${ }^{2}$ Abnormal LV compliance is a feature of longstanding poorly-controlled hypertension, ${ }^{3}$ right ventricular dysfunction, ${ }^{4}$ and pericardial disease. ${ }^{2}$ In the presence of abnormal LV relaxation and/or compliance, $L A$ contraction assumes a key role in maintaining $\mathrm{LV}$ filling. Under physiological conditions in youthful individuals, atrial contraction contributes approximately $20 \%$ of the total filling volume of the LV; whereas, in patients with LV diastolic dysfunction, LA

\section{B. A. Finegan, MB ( $\square)$}

Department of Anesthesiology and Pain Medicine,

University of Alberta, 8-120 Clinical Sciences Building,

Edmonton, AB T6G 2G3, Canada

e-mail: chassist@ualberta.ca contraction may account for over $50 \%$ of LV volume at enddiastole. ${ }^{1}$ Absence of LA contraction, as occurs with the development of atrial fibrillation, is a harbinger of heart failure and is a marker of an increased risk of premature death. ${ }^{5}$ The occurrence of abnormal LV relaxation and compliance, as a consequence of hypertensive heart disease, is one of the most common determinants of LA enlargement, poor LA contractile function, and LA dysrhythmia development. 6

It is difficult, if not impossible, to individually assess the components of LV filling in humans; however, relatively precise global estimates of diastolic function can be performed following cardiac catheterization. ${ }^{7}$ This approach is invasive, time consuming, and not without risk. In reality, both our knowledge of diastolic function in the broader clinical environment and our interest in the topic did not materialize until the introduction of pulse wave Doppler echocardiography. Pulse wave Doppler echocardiography allows the timing and amplitude of the blood flow and tissue velocity profiles within the heart to be ascertained and graphically displayed. Extensive research has determined which blood flow/tissue velocity profile patterns are considered normal and which appear to be characteristic of diastolic dysfunction. The mitral inflow velocity profile, one of the earliest patterns studied, offers a relatively simple example of the deductive reasoning underlying the evolution of Doppler derived diastolic function assessment. ${ }^{8}$ Under "normal" circumstances, flow velocity across the mitral valve is biphasic, i.e., greater during early diastole (E wave) than during subsequent atrial contraction (A wave). In the presence of mild diastolic dysfunction associated with impaired ventricular relaxation, the early diastolic passive flow velocity is reduced while the flow velocity associated with atrial contraction is enhanced (reversed E/A ratio). It is clear, however, that many factors 
other than intrinsic abnormalities of LV filling can cause an alteration from the normal E/A ratio. Indeed, this index is not only preload dependent, but also, as healthy patients age and alter their posture, mitral flow velocity profiles deviate from the expected norm. ${ }^{8,9}$ These limitations have resulted in the development of a number of other indices of diastolic dysfunction, including tissue velocity imaging and myocardial strain and strain rate imaging. ${ }^{10}$ In their elegant paper in this issue of the Journal, Couture et al. ${ }^{11}$ employed many of these indices in an effort to evaluate whether induction of anesthesia in patients with pre-existing diastolic dysfunction effected a change in myocardial diastolic performance. Not too surprisingly, they concluded that it was not possible to differentiate between the effects of anesthesia on preload and afterload and the observed alterations in the indices of diastolic dysfunction after induction.

What then is the busy clinician to take home from these findings? Is diastolic dysfunction an esoteric entity best left to cardiologists and anesthesiologists who are skilled in echocardiography and have the time and interest to pursue this arcane property of the ventricle, i.e., the ability to relax? While this may seem the appropriate course given the complex and sometimes perplexing science underpinning the diagnosis of abnormal diastolic function, it is not a path worthy of the thinking physician. Acute decompensated heart failure is the most common reason that individuals over the age of 65 in Canada are admitted to hospital. ${ }^{12}$ More than $30 \%$ of these patients have a normal or near normal ejection fraction. ${ }^{13}$ There is emerging evidence that diastolic dysfunction diagnosed prior to surgery is associated with increased morbidity and mortality. ${ }^{14}$ Risk factors for diastolic dysfunction, including poorly controlled hypertension, valvular heart disease, and overt or covert myocardial ischemia, are evident in many of our patients presenting for elective and emergency surgery. Events often associated with the perioperative period, for example, acute volume overload, ventricular tachycardia, acute exacerbation of preexisting hypertension, and/or the loss of atrial contraction, can precipitate acute pulmonary edema in this patient population. Appropriate intraoperative management strategies include judicious fluid administration to maintain preload, aggressive management of hypertension by vasodilator therapy, and beta blocker therapy to control heart rate. In the event that acute heart failure develops, furosemide should be given to the patient to induce diuresis, and positive pressure ventilation should be continued to maintain oxygenation. ${ }^{15}$

Over the last two decades, pre-emptive and reactive management of cardiac disease has dramatically improved with consequentially beneficial effects on longevity and quality of life. Ironically, this success in managing acute cardiac diseases has increased the burden of chronic heart failure due to both systolic and diastolic dysfunction. Advances in imaging and the structured formal education of anesthesiologists in the use of cardiac ultrasound offer the promise that anesthesiologists will become active participants in the search to develop appropriate management protocols for such patients undergoing surgical procedures. The anesthesiologists at Montreal Heart Institute have been pioneers in this exciting enterprise. It is now time for all of us to make our contribution.

\section{Qu'en est-il des deux autres tiers du cycle cardiaque?}

La dysfonction diastolique est un diagnostic clinique relativement récent qui est posé chez les patients dont les résultats échocardiographiques sont considérés comme correspondant à des anomalies de remplissage du ventricule gauche (VG). Le remplissage du ventricule gauche dépend de l'interaction complexe de trois processus : la relaxation du VG, la compliance du VG et la contraction de l'oreillette gauche (OG). ${ }^{1}$ La relaxation $d u$ ventricule gauche est un processus actif qui implique le recaptage du $\mathrm{Ca}^{++}$dans le réticulum sarcoplasmique par la pompe $\mathrm{Ca}^{++}$ ATPase du réticulum sarco-endoplasmique et la suppression $\mathrm{du} \mathrm{Ca}^{++}$par la cellule via l'échangeur de sodiumcalcium. L'expression et l'activité de la pompe ATPase $\mathrm{Ca}^{++}$du réticulum sarco-endoplasmique sont réduites dans le cardiomyocyte (et la fonction diastolique est modifiée) avec l'apparition de sénescence, d'hypertrophie et d'insuffisance cardiaque. La compliance du ventricule gauche est une mesure de la relation entre le développement du volume du VG et de la pression du VG pendant le remplissage du VG (dV/dp). La compliance du ventricule gauche est influencée par : 1) la présence d'une hypertrophie ventriculaire ; 2) l'état fonctionnel du ventricule droit (interaction ventriculaire diastolique directe), étant donné que les deux ventricules ont une paroi commune, le septum ; et 3) les propriétés du péricarde. ${ }^{2}$ Une compliance anormale du VG est caractéristique d'une hypertension prolongée mal contrôlée, ${ }^{3}$ d'une dysfonction du ventricule droit, ${ }^{4}$ et de maladies péricardiques. ${ }^{2}$ Lorsqu'une relaxation ou une compliance anormales du VG sont observées, la contraction de l'OG joue un rôle fondamental pour maintenir le remplissage du VG. Dans des conditions physiologiques chez des individus jeunes, la contraction auriculaire est à l'origine d'environ $20 \%$ du volume de remplissage total du VG ; par contre, chez les patients souffrant de dysfonction diastolique du VG, la contraction de l'OG peut être responsable de plus de $50 \%$ du volume du VG en télédiastole. ${ }^{1}$ L'absence de contraction de l'OG, 
qui survient lors de l'apparition de fibrillation auriculaire, est un signe avant-coureur d'insuffisance cardiaque et dénote un risque accru de mort prématurée. ${ }^{5}$ L'apparition d'une relaxation et d'une compliance du VG anormales par suite d'une maladie cardiaque hypertensive est l'un des facteurs déterminants les plus fréquents de dilatation de l'OG, de fonction contractile réduite de l'OG et de manifestation d'une dysrythmie de l'OG. $^{6}$

Il est difficile, voire impossible, d'évaluer une à une les composantes du remplissage du VG chez les humains; toutefois, des estimations globales relativement précises de la fonction diastolique peuvent être réalisées après un cathétérisme cardiaque. ${ }^{7}$ Cette approche est invasive, prend du temps, et comporte des risques. En réalité, notre connaissance de la fonction diastolique dans le contexte clinique élargi et notre intérêt pour cette question n'ont vu le jour que grâce à l'introduction du Doppler pulsé en échographie. Le Doppler pulsé permet de mesurer l'amplitude du débit sanguin en fonction du temps ainsi que de confirmer et d'afficher sous forme graphique les profils de vélocité tissulaires dans le cœur. Des recherches approfondies ont permis de déterminer quels modèles de débit sanguin/profil de vélocité tissulaire sont considérés normaux et lesquels semblent être caractéristiques d'une dysfonction diastolique. Le profil de vélocité de l'apport mitral est l'un des modèles qui a été étudié le plus tôt; il offre un exemple relativement simple du raisonnement de déduction à la base de l'évolution de l'évaluation de la fonction diastolique selon le mode Doppler. ${ }^{8}$ Dans des circonstances «normales », la vélocité du débit est biphasique au niveau de la valve mitrale, c'est-à-dire qu'elle est plus élevée en début de diastole (onde E) que pendant la contraction auriculaire qui suit (onde A). Lorsqu'il y a une dysfonction diastolique associée à une relaxation et/ou compliance ventriculaires affaiblies, la vélocité du débit passif diastolique initial est réduite tandis que la vélocité du débit associée à la contraction auriculaire est renforcée (ratio E/A inversé). Toutefois, il est évident que plusieurs facteurs autres que les anomalies intrinsèques du remplissage du VG peuvent aussi provoquer des modifications du ratio E/A normal. En effet, cet indice dépend non seulement de la précharge; en plus, tandis que des patients sains vieillissent et changent leur posture, les profils de vélocité du flux mitral tendent à dévier de la norme attendue. ${ }^{8,9}$ Ces limites ont généré l'élaboration de plusieurs autres indices de dysfonction diastolique, notamment l'imagerie de la vélocité tissulaire et l'imagerie de la déformation myocardique et du taux de déformation. ${ }^{10}$ Dans leur article intéressant dans ce numéro du Journal, Couture et coll. ${ }^{11}$ ont utilisé plusieurs de ces indices afin d'examiner si l'induction de l'anesthésie chez des patients présentant une dysfonction diastolique préexistante modifiait la performance diastolique myocardique.
Sans grande surprise, les auteurs ont conclu qu'il était impossible d'établir une distinction entre les effets de l'anesthésie sur la précharge et la postcharge et les modifications observées dans les indices de dysfonction diastolique après l'induction.

Que faut-il que le clinicien occupé retienne alors de ces résultats ? La dysfonction diastolique est-elle une entité ésotérique qu'il vaut mieux laisser aux cardiologues et anesthésiologistes qui savent utiliser l'échographie et ont le temps et l'intérêt nécessaires pour explorer cette propriété obscure du ventricule - sa capacité de relaxation? Bien que cela semble être la solution adéquate étant donné la science complexe, voire déroutante qui soutient le diagnostic d'une dysfonction diastolique anormale, cette voie n'est pas digne d'un médecin qui réfléchit. Au Canada, l'insuffisance cardiaque décompensée aiguë constitue la raison numéro un d'admission à l'hôpital de patients âgés de plus de 65 ans. ${ }^{12}$ Plus de $30 \%$ des patients admis présentent une fraction d'éjection normale ou quasi normale. ${ }^{13}$ De nouvelles données probantes suggèrent qu'une dysfonction diastolique détectée avant la chirurgie est associée à une morbidité et une mortalité accrues. ${ }^{14}$ Les facteurs de risque pouvant potentiellement entraîner une dysfonction diastolique, notamment une hypertension mal contrôlée, une valvulopathie et une ischémie myocardique symptomatique ou silencieuse, sont manifestes chez bon nombre des patients qui sont admis pour subir une chirurgie programmée ou urgente. Dans cette population de patients, les événements fréquemment associés à la période périopératoire (surcharge volémique aiguë, tachycardie ventriculaire, exacerbation aiguë d'une hypertension préexistante, et/ou perte de la contraction auriculaire) peuvent précipiter l'apparition d'un œdème pulmonaire aigu. Parmi les stratégies de prise en charge peropératoire adaptées, citons une administration liquidienne judicieuse pour maintenir la précharge, une prise en charge agressive de l'hypertension avec un traitement vasodilatateur, et un traitement à base de bêtabloquants pour contrôler la fréquence cardiaque. Si une insuffisance cardiaque aiguë survient, il faut administrer du furosémide au patient afin de provoquer la diurèse, et la ventilation à pression positive doit être poursuivie afin de maintenir l'oxygénation. ${ }^{15}$

$\mathrm{Au}$ cours des deux dernières décennies, la prise en charge préventive et réactive des maladies cardiaques s'est significativement améliorée, entraînant des effets bénéfiques en matière de longévité et de qualité de vie. Toutefois, ironiquement, cette prise en charge améliorée des maladies cardiaques aiguës a aussi accru la charge que représente l'insuffisance cardiaque aiguë provoquée aussi bien par la dysfonction systolique que diastolique. Les progrès réalisés en imagerie et la formation structurée des anesthésiologistes en matière d'échographie cardiaque sont des signes prometteurs de l'engagement futur des anesthésiologistes 
dans les recherches menées pour élaborer des protocoles de prise en charge adaptés pour de tels patients devant subir une chirurgie. Les anesthésiologistes du l'Institut de cardiologie de Montréal ont été des précurseurs dans ce projet captivant. Il est temps maintenant que nous tous apportions notre contribution.

Conflicts of interest None declared.

\section{References}

1. Garcia MJ. Left ventricular filling. Heart Fail Clin 2008; 4: 47-56.

2. Morris-Thurgood JA, Frenneaux MP. Diastolic ventricular interaction and ventricular diastolic filling. Heart Fail Rev 2000; 5: 307-23.

3. Hare JL, Brown JK, Marwick TH. Association of myocardial strain with left ventricular geometry and progression of hypertensive heart disease. Am J Cardiol 2008; 102: 87-91.

4. Goldstein JA. Pathophysiology and management of right heart ischemia. J Am Coll Cardiol 2002; 40: 841-53.

5. Stewart S, Hart CL, Hole DJ, McMurray JJ. A population-based study of the long-term risks associated with atrial fibrillation: 20year follow-up of the Renfrew/Paisley study. Am J Med 2002; 113: 359-64.
6. Aksnes TA, Flaa A, Strand A, Kjeldsen SE. Prevention of atrial fibrillation in hypertension. Curr Hypertens Rep 2008; 10: 175-81.

7. Pirracchio R, Cholley B, De Hert S, Solal AC, Mebazaa A. Diastolic heart failure in anaesthesia and critical care. $\mathrm{Br} \mathrm{J}$ Anaesth 2007; 98: 707-21.

8. Schirmer H, Lunde P, Rasmussen K. Mitral flow derived Doppler indices of left ventricular diastolic function in a general population. The Tromso study. Eur Heart J 2000; 21: 1376-86.

9. Baldi JC, Lalande S, Carrick-Ranson G, Johnson BD. Postural differences in hemodynamics and diastolic function in healthy older men. Eur J Appl Physiol 2007; 99: 651-7.

10. Price DJ, Wallbridge DR, Stewart MJ. Tissue Doppler imaging: current and potential clinical applications. Heart 2000; 84(Suppl 2): II11-8.

11. Couture $P$, Denault $A Y$, Shi $Y$, et al. Effects of anesthetic induction in patients with diastolic dysfunction. Can $\mathrm{J}$ Anesth 2009; 56. (in press).

12. Lepage S. Acute decompensated heart failure. Can J Cardiol 2008; 24(Suppl B): 6B-8B.

13. Mandinov L, Eberli FR, Seiler C, Hess OM. Diastolic heart failure. Cardiovasc Res 2000; 45: 813-25.

14. Merello L, Riesle E, Alburquerque J, et al. Risk scores do not predict high mortality after coronary artery bypass surgery in the presence of diastolic dysfunction. Ann Thorac Surg 2008; 85: 1247-55.

15. Kumar R, Gandhi SK, Little WC. Acute heart failure with preserved systolic function. Crit Care Med 2008; 36(1 Suppl): S52-6. 